\title{
A DUAL FORM OF KURATOWSKI'S THEOREM
}

Frank Harary and William T. Tutte

(received May 11, 1964)

The celebrated criterion of Kuratowski [2] for the planarity of a graph G involves the determination of whether $G$ contains a subgraph homeomorphic to $\mathrm{K}_{5}$ or $\mathrm{K}_{3,3}$ shown in Figure 1 .
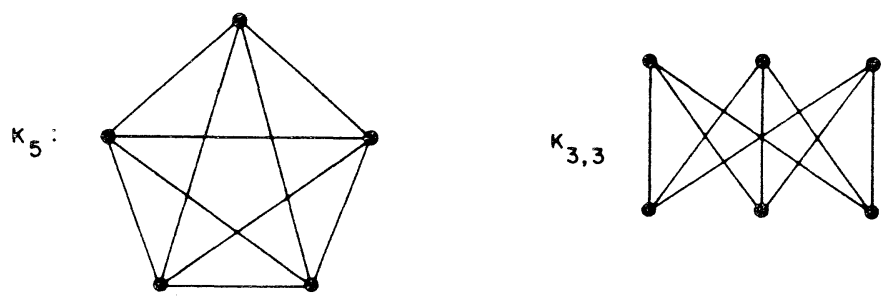

Figure 1

The well-known "Petersen graph", shown in Figure 2, looks suspiciously like $\mathrm{K}_{5}$ but nevertheless contains no subgraph homeomorphic to $\mathrm{K}_{5}$. Its nonplanar character may be confirmed by verifying the occurrence of a homeomorph of $\mathrm{K}_{3,3}$ in it.

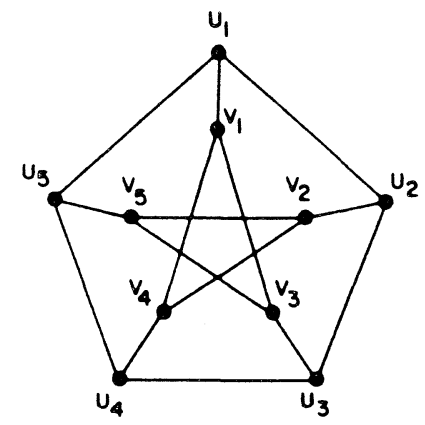

Figure 2

Our object in this note is to present a variation of Kuratowski's Theorem whereby it is possible to test a graph

Canad. Math. Bull. vol. 8, no. 1, February 1965 
for planarity by contracting comnected subgraphs into single verices. The statement of this result includes Kuratowski's criterion since homeomorphic reduction is a special case of contraction. Note that the contraction of each connected subgraph (edge) $u_{i} r_{i}$ in Figure 2 into a single vertex results in $K_{5}$.

Let $E(G)$ be the set of edges of a graph $G$ and let $V(G)$ be its vertex set. For any set $S$ of edges of $G$, we write $\bar{S}=E(G)-S$. We write $G: S$ for the subgraph $H$ of $G$ defined by $V(H)=V(G)$ and $E(H)=S$. The reduction $G \cdot S$ of $G$ to $S$ is obtained from G:S by deleting its isolated vertices.

Let $C(S)$ be the set of components of G: $\bar{S}$. The contraction $G \times S$ of $G$ to $S$ is a graph such that $V(G \times S)=C(S)$ and $E(G \times S)=S$. The ends of an edge $A$ of $G \times S$ are the members of $C(S)$ containing an end of $A$ in $G$. The notation $[u],[v], \ldots$ will be used later for the vertices of a contraction.

In the case of planar graphs it can be verified that reduction and contraction are dual operations. Even for general graphs they are dual in the sense of matroid theory [3].

A subcontraction of $G$ is a reduction of a contraction of $G$. Such a graph can also be realized as a contraction of a reduction of G. In [4], formulas are given which are general rules for inverting the orders of contractions and reductions.

THEOREM. A graph is nonplanar if and only if it has $\mathrm{K}_{5}$ or $\mathrm{K}_{3,3}$ as a subcontraction.

The usual version of Kuratowski's theorem asserts that a graph $G$ is nonplanar if and only if it contains a graph $\mathrm{H}$ homeomorphic to $\mathrm{K}_{5}$ or $\mathrm{K}_{3,3}$. If this condition is fulfilled it is clear that $G$ has a $K_{5}$ or a $K_{3,3}$ as a subcontraction.

Conversely suppose $G$ has a subcontraction $H=(G \cdot S) \times T$ which is a $\mathrm{K}_{3,3}$. Consider a vertex [v] of $\mathrm{H}$ incident with three edges $A_{1}, A_{2}$ and $A_{3}$. Then $[v]$ is a connected subgraph of $G \cdot S$. Let its vertices incident in $G$ with $A_{1}, A_{2}$ and $A_{3}$ be $v_{1}, v_{2}$ and $v_{3}$ respectively.

If $v_{1}, v_{2}$ and $v_{3}$ are distinct we can find a vertex $v$ of [v] joined to $v_{1}, v_{2}$ and $v_{3}$ by arcs $L_{1}, L_{2}$ and $L_{3}$ respect- 
ively, in $[v]$, so that no two of the $L_{i}$ intersect except for the common vertex $v$. We can make the same as sertion if two of the $v_{i}$ coincide, say $v_{1}=v_{2}$. Then $v=v_{1}=v_{2}$, and $L_{1}$ and $\mathrm{L}_{2}$ are trivial graphs, i.e., consist of one vertex only. if $v_{1}=v_{2}=v_{3}$ then $L_{3}$ is also a trivial graph.

Applying this construction to all the vertices of $H$ we can replace $H$ by a subgraph of $G \cdot S$, with vertices such as $v$, which is a homeomorph of $\mathrm{K}_{3,3}$.

A similar argument applies when $G$ has a subcontraction $\mathrm{H}=(\mathrm{G} \cdot \mathrm{S}) \times \mathrm{T}$ which is a $\mathrm{K}_{5}$. Then we may consider a vertex [v] of $H$ incident with four edges $A_{1}, A_{2}, A_{3}$ and $A_{4}$, with ends $v_{1}, v_{2}, v_{3}$ and $v_{4}$ (in G) belonging to [v]. It may be possible to find a vertex $v$ of $[v]$ which can be joined to $\mathrm{v}_{1}, \mathrm{v}_{2}, \mathrm{v}_{3}$ and $\mathrm{v}_{4}$ by arcs which meet only at $\mathrm{v}$. If this can be done for all 5 vertices of $H$, then $G$ contains a homeomorph of $\mathrm{K}_{5}$.

In the remaining case we may suppose that in one vertex [v] of $H$ the vertices $v_{i}$ can be joined up as in Figure 3. In this figure, $w_{1}, w_{2}, w_{3}$ and $w_{4}$ represent the other vertices of $\mathrm{H}$, to be considered here as connected subgraphs of $G \cdot S$.

Let $A$ be one of the edges of the arc $u_{1} u_{2}$. Let $S_{1}$ be the set obtained from $S$ by deleting the edges $w_{1} w_{3}$ and $w_{2} w_{4}$. Call $\mathrm{T}_{1}$ the set obtained from $\mathrm{T}$ by deleting the edges $\mathrm{w}_{1} \mathrm{w}_{3}$ and $\mathrm{w}_{2} \mathrm{w}_{4}$ and then adjoining $A$. Clearly $\left(G \cdot \mathrm{S}_{1}\right) \times \mathrm{T}_{1}$ is a $\mathrm{K}_{3,3}$, completing the proof of the theorem.

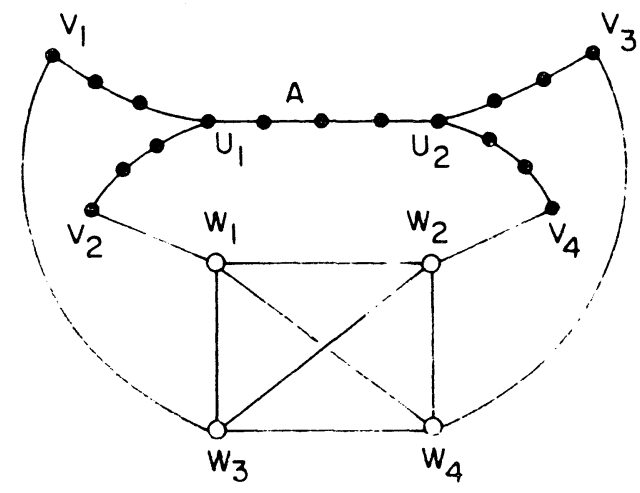

Figure 3 
We have illustrated this theorem arlier by applying it to the Petersen graph shown in Figure 2. Note that the following stronger statement may be made: A graph is non planar if and only if it has $\mathrm{K}_{5}$ or $\mathrm{K}_{3,3}$ as a contraction.

The above considerations may perhaps be relevant to the problem of extending Kuratowski's Theorem to surfaces other than the sphere. I. N. Kagno [1] has given incomplete lists of minimal graphs not realizable in the projective plane and the torus. Here the term "minimal" is used in the sense that G is not realizable in $S$ but all its subgraphs are. Perhaps we should replace the word "subgraph" by "subcontraction" in this definition. This would presumably make the list of minimal graphs, if finite, shorter.

\section{REFERENCES}

1. I. N. Kagno, The mapping of graphs on surfaces, J. Math. Phys. 16 (1937) 46-75.

2. K. Kuratowski, Sur le problème des courbes gauches en topologie, Fund. Math. 15 (1930) 271-283.

3. W. T. Tutte, Matroids and graphs, Trans. Amer. Math. Soc. 90 (1959) 527-552.

4. W. T. Tutte, On the problem of decomposing a graph into $\mathrm{n}$ connected factors, J. London Math. Soc. 36 (1961) $221-230$.

University of Michigan

and

University of Waterloo 